\title{
Low self-esteem and the formation of global self-performance estimates in emerging adulthood
}

Marion Rouault ${ }^{1,2, *}$, Geert-Jan Will ${ }^{3, *}$, Stephen M. Fleming ${ }^{4,5,6}$ and Raymond J. Dolan ${ }^{4,5}$

${ }^{1}$ Institut Jean Nicod, Département d'études cognitives, ENS, EHESS, CNRS, PSL University, 75005 Paris, France.

${ }^{2}$ Laboratoire de neurosciences cognitives et computationnelles, Département d'études cognitives, ENS, INSERM, PSL University, 75005 Paris, France.

${ }^{3}$ Department of Clinical Psychology, Utrecht University, Utrecht, The Netherlands.

${ }^{4}$ Wellcome Centre for Human Neuroimaging, University College London, London, UK.

${ }^{5}$ Max Planck UCL Centre for Computational Psychiatry and Ageing Research, University College London, London, UK.

${ }^{6}$ Department of Experimental Psychology, University College London, 26 Bedford Way, London WC1H $0 A P, U K$.

${ }^{*}$ Equal contribution

Correspondence: marion.rouault@gmail.com

Keywords: metacognition, mental health, confidence, self-esteem

Number of figures: 4

Number of supplementary figures: 2

Number of supplementary tables: 3 


\begin{abstract}
High self-esteem, an overall positive evaluation of self-worth, is a cornerstone of mental health. Previously we showed that people with low self-esteem differentially construct beliefs about momentary self-worth derived from social feedback. However, it remains unknown whether these anomalies extend to constructing beliefs about self-performance in a non-social context, in the absence of external feedback. Here, we examined this question using a novel behavioral paradigm probing subjects' self-performance estimates with or without external feedback. We analyzed data from young adults $(\mathrm{N}=57)$ who were selected from a larger community sample $(\mathrm{N}=2,402)$ on the basis of occupying the bottom or top $10 \%$ of a reported self-esteem distribution. Participants performed a series of short blocks involving two perceptual decision-making tasks with varying degrees of difficulty, with or without feedback. At the end of each block, they had to decide on which task they thought they performed best, and gave subjective task ratings, providing two measures of selfperformance estimates. We found no robust evidence of differences in objective performance between high and low self-esteem participants. Nevertheless, low self-esteem participants consistently underestimated their performance as expressed in lower subjective task ratings. These results provide an initial window onto how cognitive processes underpinning the construction of self-performance estimates across different contexts map on to global dispositions relevant to mental health, such as self-esteem.
\end{abstract}


A positive view of the self is a crucial determinant of mental health ${ }^{1}$. Low self-esteem has been associated with a number of psychiatric conditions, particularly those of anxious and depressive nature ${ }^{2}$. People form beliefs about themselves and their abilities ("self-beliefs") across many levels of abstraction, from local confidence in individual decisions to self-performance estimates on a particular task, up to global estimates about their own worth as expressed in reports of self-esteem. Having positive beliefs about self-worth (i.e., high self-esteem) is associated with a stronger ability to successfully deal with prospective situations, including how one deals with day-to-day challenges ${ }^{3}$. For instance, people with low self-esteem are often faster to disengage from a task in response to failure than those with high self-esteem ${ }^{4}$. Despite the recognized importance of self-beliefs for mental health, surprisingly little is known about the precise cognitive building blocks of self-beliefs, and their relationship with self-esteem ${ }^{5}$.

Recent work examining the construction of momentary self-worth from social feedback ${ }^{6,7}$ has started to uncover the formation of self-beliefs in a social context. Here low self-esteem participants were slower to update beliefs about how much others liked them, and faster to update momentary feelings of self-worth in response to social feedback. These findings provide initial evidence of a differential construction of self-beliefs being tied to a more global, stable construct such as self-esteem ${ }^{6,7}$. However, it remains unclear whether this is a specific idiosyncrasy of how low self-esteem individuals construct self-worth from social feedback or, alternatively, whether low self-esteem individuals have a domain-general bias when forming appropriate self-beliefs that extend to other, non-social, contexts. One possibility is that individuals with low self-esteem may maintain a negative self-view by consistently underestimating their abilities despite performing as well as those with high self-esteem, indicating a disconnection between a "local" self-evaluation on a given task and a "global" self-evaluation such as self-esteem.

To test this hypothesis, here we examined the formation of subjective self-performance estimates in participants with high and low self-esteem, in contexts with and without explicit feedback about performance. We leveraged a recently developed behavioral paradigm probing the formation of subjective self-performance estimates ${ }^{8,9}$. The key finding from this previous work is that decision confidence is a key factor contributing to the formation of self-performance estimates in the absence 
of feedback, a situation that echoes many real-life settings. We observed that decision difficulty, fluctuations in decision accuracy, and whether participants received feedback about their decisions all impacted their self-performance estimates. The present study employed this protocol to ask whether such subjective self-performance estimates, formed over the scale of minutes, relate to self-esteem. We assume the latter is a global estimate formed across longer timescales of months or years. This question is important as a better understanding of neurocognitive building blocks underpinning constructs relevant to mental health, such as self-esteem, is a key step in preventing disorders that are linked to altered self-esteem, the key example being depression ${ }^{10,11}$. We previously proposed a theoretical framework in which self-esteem may act as a global prior for generating self-performance estimates on a given task ${ }^{12}$. Specifically, under such a hierarchical framework of metacognitive evaluation - spanning decision confidence formed at a local level to self-esteem at a global level - we would expect self-esteem to provide a global context or prior for how self-performance estimates are formed on a given task ${ }^{5}$.

We capitalized on a large dataset from a well-characterized community sample of adolescents and emerging adults ( $\mathrm{N}=2,402$; aged 14 to 24 at first measurement) that reported on their self-esteem across 1-3 measurements spanning 4.5 years. We selected low and high self-esteem participants (aged 18-25) from the larger sample based on consistently scoring within the bottom, or top, $10 \%$ of a selfesteem distribution so as to maximize power for detecting individual differences due to self-esteem ${ }^{7}$. Participants performed short blocks of two interleaved perceptual tasks and at the end of each block they then selected the task on which they considered they had performed best, and provided a subjective ability rating about each task. These two measures enable a window onto subjective selfperformance estimates ${ }^{8}$.

Consistent with previous findings, we found participants underestimated their own performance in the absence of feedback, despite performing equivalently in situations with and without feedback. Participants with low self-esteem underestimated their performance compared to those with high self-esteem, despite task performance being similar in the two groups. We discuss the findings within a framework in which local metacognitive variables, such as decision confidence, influence the construction and maintenance of global self-esteem across longer times-scales. 


\section{Results}

\section{An experimental protocol probing the formation of self-performance estimates}

To investigate the impact of self-esteem on self-performance estimates, participants $(\mathrm{N}=57)$ engaged in 30 short learning blocks (4 to 20 trials) of two randomly interleaved visual discrimination tasks signaled by two arbitrary color cues (Fig. 1c). Each task required a perceptual discrimination judgment as to which of two boxes contained a higher number of dots (Fig. 1c). Two factors controlled task characteristics: task difficulty (either easy or difficult according to dot difference between boxes), and receipt of either veridical feedback (correct, incorrect) or no feedback about performance on each perceptual choice (Fig. 1b). This factorial design resulted in six possible task pairings for learning blocks (Fig. 1a). For example, an Easy-Feedback task could be paired with a Difficult-Feedback task, or a Difficult-Feedback task could be paired with a Difficult-No-Feedback task, and so forth. At the end of each block, participants selected the task on which they believed they performed better (Task choice) and were rewarded on the basis of their performance on the chosen task (see Methods). They additionally provided a subjective rating of self-performance on each of the two tasks on a continuous scale (Task ratings) (Fig. 1a). A short break ensued before the next learning block started, when two new color cues indicated two new tasks. The two end-of-block measures, namely task choice and task ratings, provided proxies for self-performance estimates. In this way the design allowed us to compare self-performance estimates in participants with high or low self-esteem. 
a

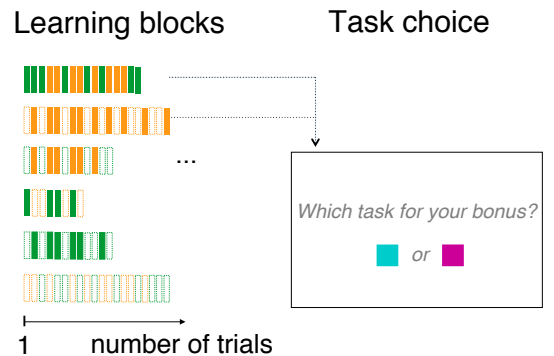

C

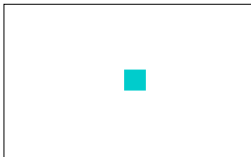

Task cue $1200 \mathrm{~ms}$

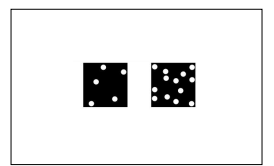

Stimuli $300 \mathrm{~ms}$
Task rating
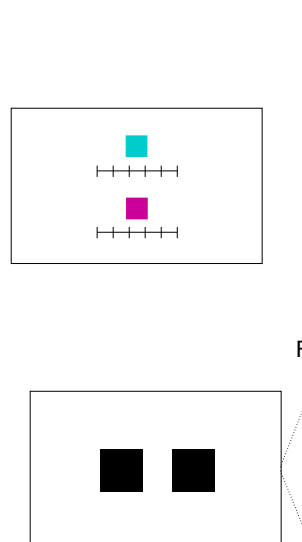

Response untimed b

Feedback No Feedback

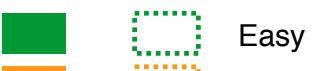

Difficult

Figure 1. Experimental design probing the construction of self-performance estimates adapted from ${ }^{8}$. a) Participants performed short learning blocks of randomly alternating trials from two tasks (between 2 and 10 trials per task). At the end of each block, participants were asked to select the task on which they thought they had performed best (Task choice), as well as rated their overall ability at each task (Task rating). A new block ensued with two new color cues indicating two new tasks. b) Each task required perceptual choices as to which of two boxes contained more dots. Trials were either easy or difficult according to the numerical dot difference between the left and right boxes. Following their response participants either received veridical feedback (correct, incorrect) about their perceptual judgment, or no feedback. These four conditions resulted in six possible task pairings as displayed in a. c) Each trial consisted in a perceptual judgment as to which of two boxes contained a higher number of dots, followed or not by provision of feedback.

\section{Self-esteem and self-performance estimates}

We first examined whether high and low self-esteem participants differed in terms of objective performance on the perceptual tasks, conditional on provision of feedback or not, and on task difficulty. We performed a $2 \times 2 \times 2$ repeated-measures ANOVA on objective performance with two within-subject factors (Feedback and Difficulty), and with self-esteem group as a between-subject factor (see Methods). First, we replicated our previous findings showing that participants performed better when tasks were easier (main effect of Difficulty, $\mathrm{F}(1,56)=472.7, p<.001$ ), but without a difference in performance in the presence or in the absence of feedback (Fig. S1) (main effect of Feedback, $\mathrm{F}(1,56)=0.622, p=.434)$. High $(\mathrm{N}=28)$ and low $(\mathrm{N}=29)$ self-esteem participants did not differ in performance (main effect of Self-Esteem, $\mathrm{F}(1,56)=1.675, p=.201$ ). We found no significant interactions, except for an interaction between Difficulty and Self-Esteem $(\mathrm{F}(1,56)=5.174, p=.027)$ driven by slightly worse performance on easy tasks in the low self-esteem group (Table S1). However, 
in the absence of a main effect of Self-Esteem on performance, it is likely any difference in selfperformance estimates between self-esteem groups arose out of differences in formation of selfperformance estimates, rather than a by-product of systematic differences in objective performance between groups.

Next, we examined the construction of self-performance estimates in our perceptual tasks. We again applied the same $2 \times 2 \times 2$ repeated-measures ANOVA, this time to task choices and task ratings. For our first measure of self-performance estimates, task choice, we replicated our prior work showing participants selected easy tasks as compared to difficult tasks more often at the end of blocks (main effect of Difficulty $\mathrm{F}(1,56)=108.8, p<.001)$. Participants were also more likely to select tasks that provided feedback, compared to those that did not (main effect of Feedback $\mathrm{F}(1,56)=93.8, p<.001$ ). There was a trend-level interaction between Difficulty and Feedback $(\mathrm{F}(1,56)=3.81, p=.056)$, in accordance with previous findings showing an interaction in only some datasets (Fig. S1) ${ }^{8}$. This we assume reflects variability in how sensitive participants are to difficulty relative to feedback receipt. We found no main effect of Self-Esteem on task choice $(\mathrm{F}(1,56)=.295, p=.59)$ and no significant interactions between Self-Esteem and other experimental factors (all $p>.33$ ), meaning that task choices were most likely driven by experimentally manipulated factors as opposed to (task-unrelated) selfesteem. We acknowledge here that task choices are coarse measures (i.e., binary choices) as compared to more fine-grained parametric task ratings, possibly explaining an absence of effects (see also Discussion).

Finally, we analyzed our second measure of self-performance estimates: subjective task ability ratings. Consistent with previous findings ${ }^{8}$, we found a main effect of Difficulty $(F(1,56)=211.7$, $p<.001)$ and of Feedback $(\mathrm{F}(1,56)=139.9, p<.001)$ on task ratings, together with a significant interaction between these factors $(\mathrm{F}(1,56)=35.6, p<.001)$. These results indicate that participants rated their self-performance lower in the absence of feedback, an effect exacerbated for easy as compared to difficult tasks (Fig. 2 and S1). Crucially we observed a main effect of Self-Esteem on task ratings $(\mathrm{F}(1,56)=5.92, p=.018)$, reflecting the fact that participants with low self-esteem reported lower selfperformance estimates for both difficult and easy tasks as well as tasks with and without feedback, 
despite their objective task performance being equivalent to participants with high self-esteem (Table S3).

Study participants were recruited on the basis of their self-esteem score at the time of inclusion in the database ("past" self-esteem level). We also assessed their self-esteem level at the time they performed the perceptual learning tasks ("current" self-esteem level) in order to create the groupings for the analyses reported above. Past self-esteem scores at the time of recruitment correlated with current self-esteem scores at the time of testing $(\rho(59)=.74, p<\mathrm{e}-10)$. Nevertheless, to examine the robustness of our findings, we reproduced all our analyses but now based on past self-esteem groupings instead of current self-esteem groupings. Critically we found virtually identical results (Table S4-S6 and Supplementary Results), indicating that our behavioral task interacts with selfesteem status in a stable manner. In particular, participants with lower self-esteem at the time of recruitment continued to provide diminished subjective task ratings at the time of testing, despite an unaffected objective performance (Table S6).
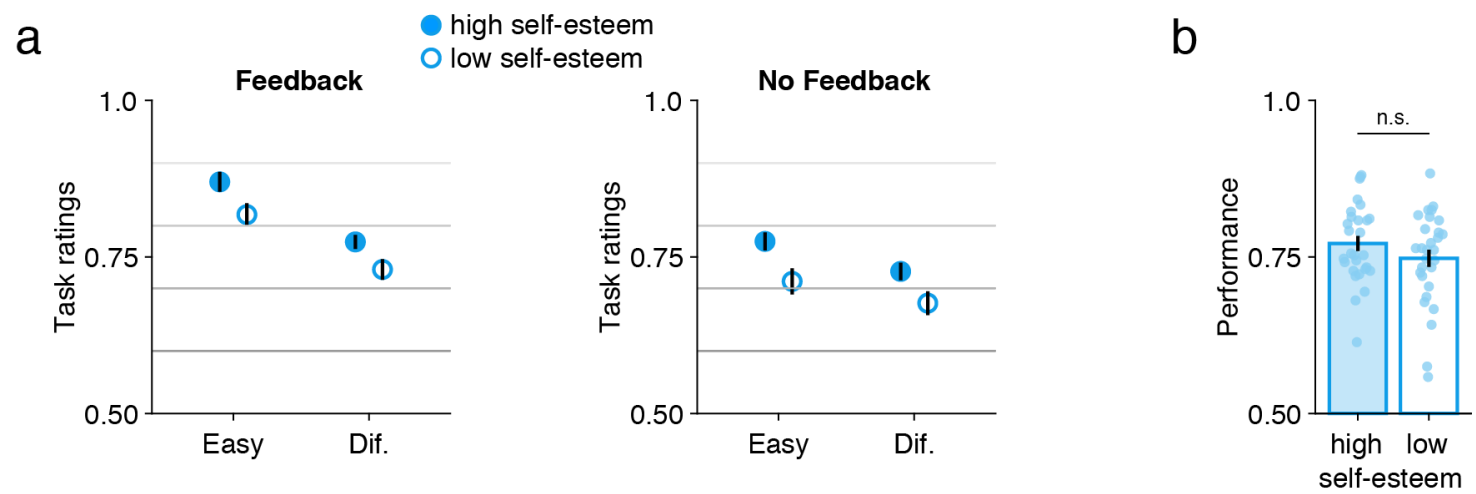

Figure 2. a) A $2 \times 2 \times 2$ repeated measures ANOVA revealed a main effect of self-esteem status on task ratings, indicating lower self-performance estimates in participants with a low self-esteem status. Difficulty (Easy, Diff) and Feedback (Feedback, No Feedback) were within-subject factors and Selfesteem was a between-subject factor (see Methods and Results). Circles and error bars represent mean and SEM across participants ( $N=28$ with high self-esteem and $N=29$ with low self-esteem). $b$, Average objective performance across all conditions for high and low self-esteem groups separately. Bar and error bars indicate mean and SEM across participants and dots indicate individual data points. $n . s$., not significant (two sample t-test, $t_{55}=1.29, p=.20$ ).

\section{Characterization of self-performance estimates independently of self-esteem}

Having shown that self-esteem is linked to self-estimates in our task, we next characterized how participants form self-performance estimates as a function of experimental factors. Extending upon our previous study ${ }^{8}$, our experimental design, with variable block lengths, allowed us to 
characterize how experimental factors explain variation in subjective task ratings, and examine if other previous findings replicate. In a first analysis, we reasoned that, even for a fixed difficulty level, there would be fluctuations in objective performance from block to block due to variability inherent to perceptual decision-making. To investigate whether participants were sensitive to such fluctuations when they provided self-performance estimates, we performed regression analyses predicting task choice and task ratings from the difference in objective performance between tasks (Fig. 3; see Methods). In a second analysis, we examined the influence of learning duration on expression of selfperformance estimates (Fig. 4; see Methods). In both these series of analyzes, when including selfesteem as an additional regressor, we consistently found no modulation of the pattern of results, together with no contribution of self-esteem in addition to the effect of the difference in performance (task choices: all $p \mathrm{~s}>.12$; task ratings: all $p \mathrm{~s}>.09$ ) or learning duration (task choices: all $p \mathrm{~s}>.10$; task ratings: all $p \mathrm{~s}>.054)$ on self-performance estimates. Therefore, we hereafter pooled behavioral data across self-esteem groups in order to increase statistical power ( $\mathrm{N}=57$ participants).

First, collapsing across self-esteem groups, we found a significant effect of a difference in performance between tasks on end-of-block task choices (all task pairings $\beta>4.51$, all $p<1.35 \mathrm{e}-07$ ), except for when an Easy-No-Feedback task was paired with a Difficult-No-Feedback task $(\beta=.28$, $p=.50$ ) (Fig. 3a). When introducing each participant's individual self-esteem score as an additional regressor, the results remain unchanged, demonstrating that objective performance influences selfperformance estimates irrespective of self-esteem levels. Task choices were sensitive to fluctuations in performance for all task pairings $(\beta=1.05, p=1.4 \mathrm{e}-7)$ except for when an Easy-No-Feedback task was paired with a Difficult-No-Feedback task $(\beta=.08, p=.50)$.

Using a similar approach, we uncovered a significant effect of differences in performance between tasks on end-of-block task ratings (Fig. 3b) (all task pairings $\beta>.054, p<.0023$ ), meaning that the larger the difference in objective performance between tasks, the larger the difference in task ratings (irrespective of self-esteem). When introducing self-esteem as a regressor, we again found significant effects of fluctuations in performance on subjective performance ratings irrespective of self-esteem levels (all $\beta>.016$, all $p \mathbf{s}<.0023$ ). Taken together, these findings indicate that participants' 
end-of-block self-performance estimates were sensitive to fluctuations in objective difficulty, the presence of feedback, and fluctuations in task performance.

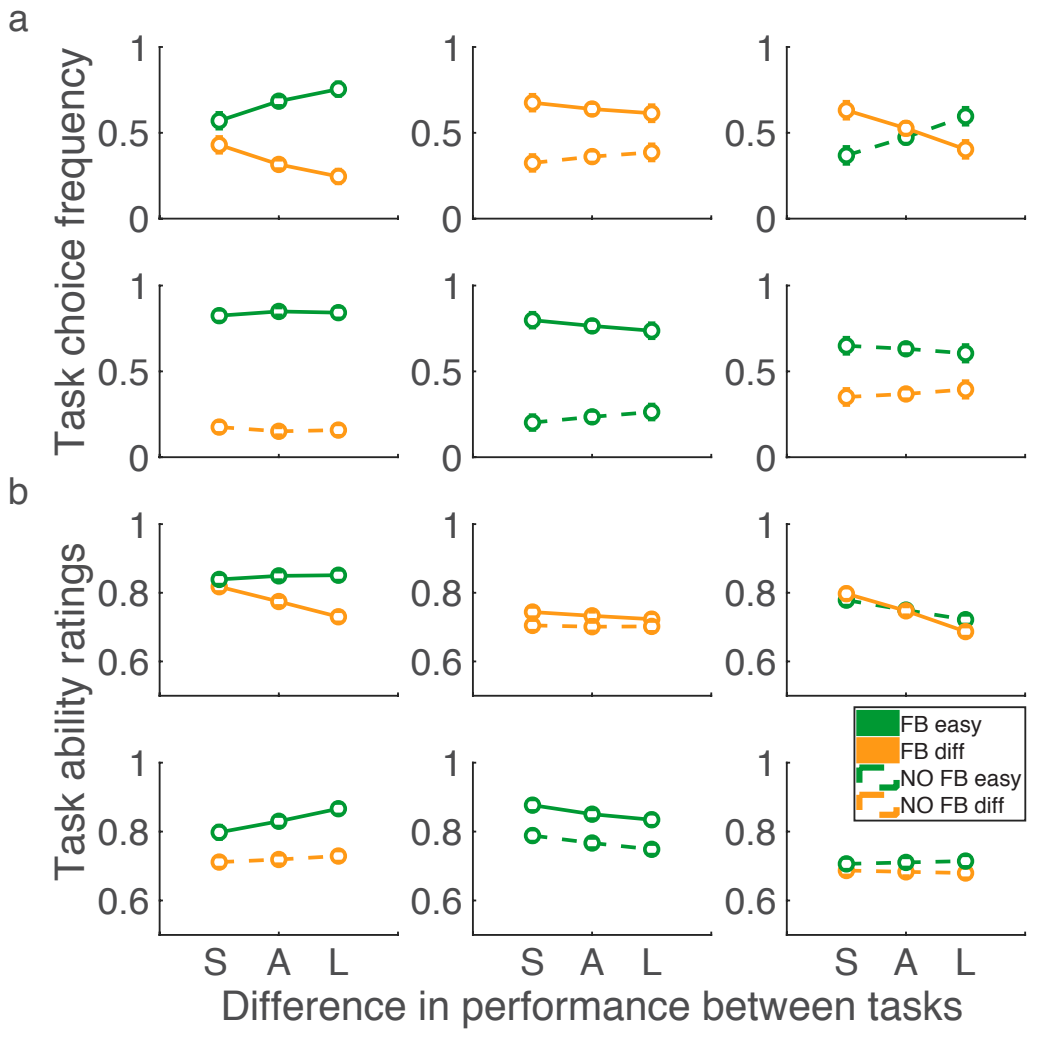

Figure 3. Participants' self-performance estimates were sensitive to difficulty level, objective performance and feedback presence. Self-performance estimates were measured as (a) task choice and (b) task ability ratings, each visualized here as a function of difference in performance between tasks (S: small, A: average, L: large difference in performance). Green (resp. orange) indicates easy (resp. difficult) tasks. Dotted lines (resp. full lines) indicate tasks without feedback (resp. with feedback). Error bars indicate S.E.M. across participants $(N=57)$.

Second, we examined the impact of learning duration on self-performance estimates. Consistent with previous findings ${ }^{8}$, regression analyses confirmed no significant effect of learning duration (number of trials per block) on end-of-block task choices (for all task pairings, all $\beta<.07$, all $p>$.11) (Fig. 4a). When we add individual self-esteem scores as a regressor, we continued to find no influence of learning duration on task choices (for all task pairings, all $\beta<.21$, all $p>.11$ ) and no influence of self-esteem (for all task pairings, all $\beta<.20$, all $p>.10$ ). Similarly, we found no effect of learning duration on end-of-block task ratings (Fig. 4b) (for all task pairings, all $\beta<.012$, all $p>0.16$ ), except for when an Easy-Feedback task was paired with an Easy-No-Feedback task $(\beta=-0.018$, $p=.034)$. When additionally including self-esteem as a regressor, we again found no effect of learning 
duration (for all task pairings, all $\beta<.012$, all $p>.16$ with the same exception, $\beta=-0.018, p=.034$ ) or self-esteem (for all task pairings, all $\operatorname{abs}(\beta)<.015$, all $p \mathrm{~s}>.054$ ) on task ratings. Together these findings indicate that participants' self-performance estimates were insensitive to task duration, suggesting participants relatively rapidly form an estimate of their expectations of success at the beginning of each block of trials.

a

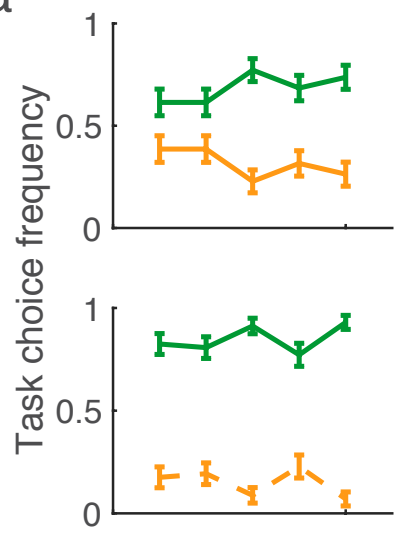

b

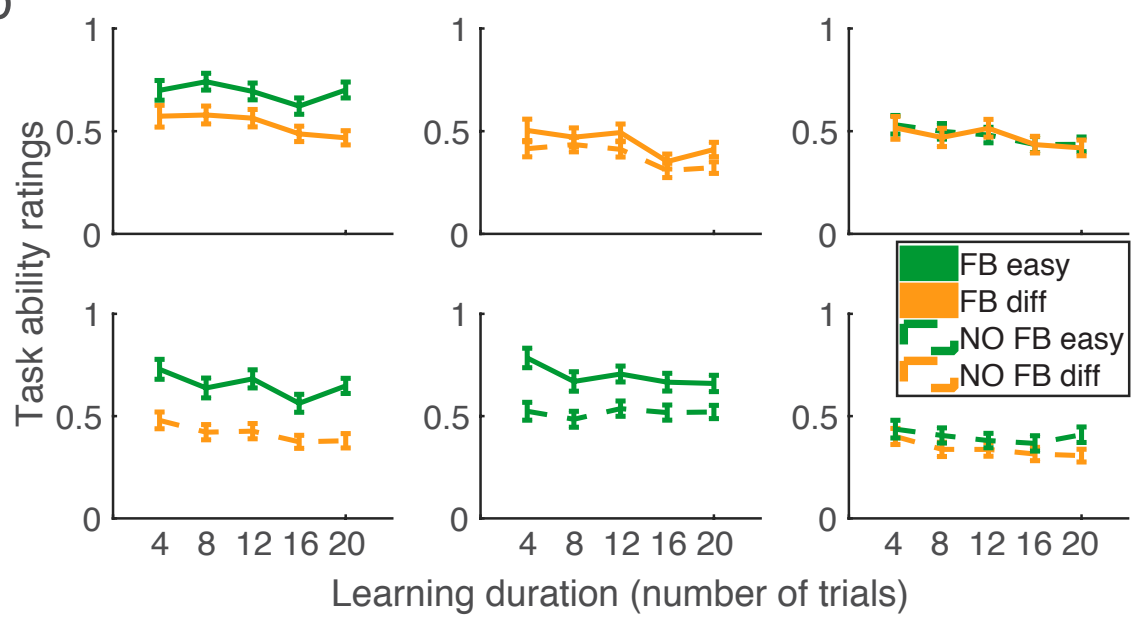

Figure 4. Self-performance estimates measured as end-of-block task choices (A) or task ratings (B) as a function of learning duration (number of trials per block) for the six possible task pairings. We found little to no effects of learning duration on self-performance estimates. Error bars indicate S.E.M. across participants ( $N=57$, pooled across self-esteem groups). 


\section{Discussion}

Humans construct beliefs about themselves, and their abilities, across many levels of abstraction, encompassing not only global constructs such as self-esteem but also self-performance estimates on a given task ${ }^{5}$. Having a favorable appraisal of oneself is a key component to mental wellbeing ${ }^{1,2}$. We previously proposed a hierarchical framework in which self-esteem acts as a global prior in self-performance estimates for a given task ${ }^{12}$. Here, we sought behavioral evidence that bears on this framework by relating self-esteem, a global construct, to subjective self-performance estimates created during tasks performed over a short temporal duration. To do this we leveraged a perceptual task for which we previously characterized how participants provide self-performance estimates ${ }^{8}$.

We replicated our previous findings showing participants' self-performance estimates are sensitive to task difficulty, the presence or absence or feedback, and fluctuations in objective task performance. We further showed that participants with low self-esteem provide lower subjective task ratings than those with high self-esteem, in the absence of a main effect of self-esteem on objective performance. This disconnect between objective performance and its subjective evaluation may be relevant for a better understanding of psychiatric disorders characterized by distortions in selfevaluation, which we will further discuss below.

An important feature of our results is the absence of systematically lower performance in participants with low self-esteem. This indicates that the difference in global self-performance estimate stemmed from a metacognitive bias, as opposed to a rational updating of confidence as a function of objectively lowered performance. This also argues against a case that lower subjective task confidence acted as a self-fulfilling prophecy, as this would have led us to also observe an overall lower performance in turn. This is a key point as previous reports have indicated that low self-esteem individuals also underestimate their performance on familiar tasks, but it has remained unclear whether this is a consequence of negative self-beliefs, or else due to negative experiences with the task at hand (for a review, see ${ }^{13}$ ). In the present study, a lack of a clear influence of low self-esteem on performance may reflect participants' having limited experience with the perceptual task. We leveraged the fact that presumably nobody had encountered the current perceptual task before in order 
to minimize prior beliefs about expected performance, thereby allowing us to isolate a 'pure' effect of self-esteem. Instead, had it been a memory task for instance, participants might have retrieved and relied on general prior beliefs about their memory abilities ${ }^{14}$. The type of perceptual task we exploit is also likely to preclude influences seen in other cognitive domains, such as mathematics in school and mathematics anxiety ${ }^{15}$, or pervasive social effects such as stereotype threat (a perceived risk of confirming negative stereotypes about abilities associated with one's social group) that are thought to influence subsequent performance ${ }^{16,17}$. Therefore, it is possible relationships between these levels may be even tighter in real-life metacognitive evaluations.

Although we cannot draw strong conclusions from non-significant findings, the lack of statistical interactions between self-esteem and experimental factors (feedback presence, difficulty level) on self-performance estimates indicates participants with low self-esteem were not only impaired in building self-performance estimates in the absence of feedback alone, which would have indicated a specific impairment in relying on internal confidence when no external validation is available. Instead, participants with low self-esteem displayed a general underestimation of their performance as seen in subjective task ratings, independently of feedback condition, and difficulty level. This result is consistent with a recent study showing that overall confidence on a perceptual task was associated with self-esteem score, in the absence of a relationship between self-esteem and metacognitive sensitivity (i.e., how well confidence tracks performance in the absence of feedback) ${ }^{18}$. Other studies have reported that self-esteem affects sensitivity to feedback, suggesting that high selfesteem may act as a 'buffer' against negative feedback ${ }^{19}$. Low self-esteem participants were found to provide self-worth ratings that are more sensitive to social evaluative feedback ${ }^{7}$ or achievement feedback ${ }^{19}$, although the type of feedback and task temporality were substantially different from those of the current study.

Many clinical and subclinical psychiatric symptoms are associated with alterations to various aspects of metacognition ${ }^{20,21}$. Low self-esteem is a predictor of mental health disorders, particularly those associated with negative experiences as expressed in anxious and depressive symptoms ${ }^{1}$. Importantly, the participants in our sample did not have a formal mental health diagnosis, providing stronger evidence that the observed associations between self-esteem and lower subjective 
performance ratings are likely to be explained by low self-esteem alone, rather than factors associated with patient status, such as stigma, the impact of therapy or medication. Similarly, a previous study reported that self-esteem predicted overall confidence on a perceptual task in an online general population sample, even after controlling for depressive symptoms ${ }^{18}$. As is typically the case, here and in our previous study ${ }^{7}$, self-esteem groups differed on trait anxiety, state anxiety, depression and social anxiety, reflecting existing associations between self-esteem levels and these symptoms. In another study using a dimensional approach, we identified lower levels of trial-by-trial decision confidence in subclinical participants who displayed higher scores on an "anxious-depression" transdiagnostic dimension ${ }^{22}$. It is unknown whether this alteration in decision confidence might generalize to more global aspects of metacognition, such as the self-performance estimates measured here. However, to the extent that self-esteem is related to negative affective symptoms, the present results showing a link between low self-esteem and lower subjective task ratings provides initial evidence this may indeed be the case. Furthermore, in previous work we have shown that global SPEs in a similar task are sensitive to trial-to-trial fluctuations in decision confidence ${ }^{8,9}$ - suggesting factors that influence baseline decision confidence are also likely to influence global metacognition.

Similarly, previous work has provided evidence that other aspects of metacognition are shifted in the context of negative affective symptoms. A previous study documented a lack of a positivity bias, meaning tendency to overweight positive as compared to negative feedback in healthy human subjects, in social anxiety when processing feedback from a social task involving giving a speech in front of judges ${ }^{23}$. To the extent that social anxiety and low self-esteem are linked, these results suggest a similar lack of a positivity bias in learning may be linked to low self-esteem. Finally, in a face discrimination task participants with high anxiety manifest different feedback-related negativity correlates following evaluative feedback, as compared to participants with low anxiety ${ }^{24}$. This indicates that anxiety might disrupt an evaluative component of performance monitoring, which we expect would extend to low self-esteem to the extent that anxiety and lowered self-esteem overlap.

Replicating ours and others' previous studies we found that task choices were sensitive to fluctuations in performance ${ }^{8,9,25}$, an effect that remained when controlling for self-esteem level. Note that unlike when making task ratings, the need to make binary task choices forces participants to 
separate between higher and lower self-performance estimates, even if self-performance on both tasks are close at the end of a block. We also replicate our previous result that learning duration did not systematically affect task choices ${ }^{8}$ and extend this finding to the case of task ratings (Fig. 4). Although here we did not measure participants' precision or confidence in their subjective task ratings, it is possible that uncertainty around expected performance decreases with learning duration, as participants have more samples to inform their self-performance estimates.

While here we investigated the formation of global self-performance estimates over the course of short learning blocks, future work is needed, particularly longitudinal measurements, to examine how global self-performance estimates develop over longer timescales ${ }^{2}$ and impact subsequent metacognitive judgments ${ }^{26}$. This can provide a window onto the formation and maintenance of global dispositions that evolve across weeks or months, such as self-esteem itself ${ }^{5}$. In the present study, participants span a limited age range and it remains unknown how the formation of experimental selfperformance estimates mirrors the maintenance and update of self-esteem across the lifespan. Some of these effects may be specific to adolescence, as previous work has shown that perceptual metacognitive sensitivity continues to mature in the 11-17 years old range ${ }^{27}$. In our sample, only a few people $(<10 \%)$ shifted their reported self-esteem sufficiently to move between the self-esteem groups over the course of a couple of years. Nevertheless, it remains to be established how malleable such self-constructs are, though the lack of interactions between self-esteem and feedback in our experiment suggests a certain degree of stability or rigidity. Under a hierarchical framework, it is plausible that higher, more global, levels are more temporally stable whereas lower levels such as local decision confidence or self-performance estimates on individual tasks may be more malleable $28,29$.

Metacognition operates across many levels of abstraction, from local confidence in individual decisions to self-performance estimates on a particular task, to global self-evaluations such as selfesteem. However, the relationships among these levels remain to be characterized. Our approach was to recruit participants from a community sample and use a task for which participants had no prior experience, academic stakes or relevance, enabling us to isolate an effect of self-esteem on selfperformance estimates from other factors typically present in patient studies. Our study therefore 
connects two of these levels of metacognition in a simple lab-based task, disconnected from real-life evaluations, finding that low self-esteem is associated with lower subjective task ratings. 


\section{Methods}

\section{Participants}

We tested 61 human participants from the Neuroscience in Psychiatry Network (NSPN) cohort $(\mathrm{N}=2,402)$ who reported on their mental health, including measures of self-esteem, across 4.5 years for 1-3 measurements ${ }^{7}$. For recruitment based on self-esteem, we used scores on the Rosenberg selfesteem scale (RSES) ${ }^{30}$. Mean RSES score of the large sample was 19.7 (on a scale of 0-30; $\mathrm{SD}=5.62$ ). We invited 184 participants with average RSES scores within the bottom decile (0-12) and top decile (27-30) of the large sample for further study and tested 53 participants (29 low self-esteem; 24 high self-esteem). To reach our target sample size of 30 participants in each group, we invited a further 51 participants whose recent RSES score was within the bottom or top decile of RSES scores and tested an additional 10 participants. Five low self-esteem participants reported being in remission from a mental health problem for at least three years at the moment of testing. The final sample consisted of 30 low self-esteem participants (mean age $=21, \mathrm{SD}=1.9 ; 18$ females) and 31 high self-esteem participants (mean age $=21, \mathrm{SD}=2.3 ; 16$ females). We matched groups based on gender and age, but not for subclinical symptoms of depression and anxiety ${ }^{7,31}$. Other inclusion criteria were applied: no current neurological or psychiatric disease, an address in London, no color-blindness and no contraindications to MRI as the participants also underwent MRI scanning (see ${ }^{7}$ ). Participants were paid 8 GBP per hour for their participation and compensated for travel expenses. They provided written informed consent according to procedures approved by the London - Westminster NHS Research Ethics Committee (15/LO/1361). Four participants were excluded for responding at chance level (2 participants, both high self-esteem), or always selecting the same rating (1 participant, low selfesteem), or failing the comprehension test of the rating scale during the practice (1 participant, high self-esteem), leaving $\mathrm{N}=57$ participants for data analysis.

\section{Experimental design}

Learning blocks. Participants performed short learning blocks that randomly interleaved two "tasks" 
identified by two arbitrary color cues (Fig. 1a). Participants were incentivized to learn about their own performance on each of the two tasks over the course of a block. Each block contained 2, 4, 6, 8 or 10 trials per task, giving 30 blocks (=360 trials) per participant, presented in a pseudo-random order. Each task required a perceptual judgment as to which of two boxes contained more dots (Fig. 1a). The difficulty level of the judgment was controlled by the difference in dot number between boxes. Any given task (as indicated by the color cue) was either easy or difficult and provided either veridical feedback or no feedback (Fig. 1b). Importantly the color cues allowed participants to identify the two tasks, but provided no information about task difficulty. These four task features provided six possible pairings of tasks in learning blocks, the order of which was randomized for each participant.

\section{Two measures of self-performance estimates.}

(1) Task choice. At the end of each block, participants were asked to choose the task for which they thought they performed best (Fig. 1a). Specifically, they were asked to report which task they would like to perform in a short subsequent "test block" in order to gain a bonus. This procedure aimed to reveal self-performance estimates, because participants should choose the task they expect to be more successful at in the test block in order to gain maximum reward. To indicate their task choice, participants responded with two response keys that differed from those assigned to perceptual decisions to avoid any carry-over effects. The subsequent test block contained six trials from the chosen task (not shown in Fig. 1). No feedback was provided during test blocks.

(2) Task ratings. After the test block, participants were asked to rate their overall performance on each of the two tasks on a rating scale ranging from $50 \%$ ("chance level") to $100 \%$ ("perfect") to obtain explicit, parametric reports of self-performance estimates (Fig. 1a). Ratings were made with the mouse cursor and could be given anywhere on the continuous scale. Intermediate ticks for percentages $60,70,80$ and $90 \%$ correct were indicated on the scale but without verbal labels. There was no time limit on perceptual choices, task choices and task ratings. After each block, participants were offered a break and could resume at any time, with 
the next learning block featuring two new tasks cued by two new colors. The present design is a modified version of the protocol from our original paper ${ }^{8}$.

Trial structure. Each block featured two tasks, with each trial starting with a color cue presented for $1200 \mathrm{~ms}$, indicating which of the two tasks will be performed in the current trial (Fig. 1). The stimuli were black boxes filled with white dots randomly positioned and presented for $300 \mathrm{~ms}$, during which time participants were unable to respond. We used two difficulty levels characterized by a constant dot difference, but the spatial configuration of the dots inside a box varied from trial to trial. One box was always half-filled ( 313 dots out of 625 positions), whereas the other contained $313+24$ dots (difficult conditions) or $313+60$ dots (easy conditions). Participants were asked to judge which box (left or right) contained more dots and the chosen box was then highlighted for $300 \mathrm{~ms}$. Afterwards, a colored rectangle (cueing the color of the current task) was presented for $1500 \mathrm{~ms}$. The rectangle was either empty (on no-feedback trials) or contained the word "Correct" or "Incorrect" (on feedback trials), followed by an ITI of $600 \mathrm{~ms}$.

\section{Statistical analyses}

To examine the influence of our experimental factors on self-performance estimates, we carried out three $2 \times 2 \times 2$ repeated measures ANOVAs on (1) objective performance (Table S1), (2) task choice (Table S2) and (3) task ratings (Table S3). Our factors were Feedback (present vs. absent), Difficulty (easy vs. difficult) as within-subject factors and self-esteem level (high vs. low) as a between-subject factor. Because task choice frequencies are proportions, they were transformed using a classic arcsine square-root transformation before entering the ANOVA. Note that we reproduced these analyses based on past self-esteem status (as per recruitment) instead of current self-esteem status (on testing session) and found virtually identical results (Tables S4-S6).

Since objective performance naturally fluctuates even for a fixed difficulty level, due to noise in perceptual systems, we examined whether participants had some insight about these fluctuations. We additionally examined whether participants' self-performance estimates reflected fluctuations in objective performance on a given learning block over and above variations in difficulty level. For each 
of the six pairings, we analyzed task choice and task ratings as a function of the difference in performance between tasks for each participant (as in ${ }^{8}$ ) (Fig. 2). To quantify these effects, we performed a logistic (resp. linear) regression to further quantify the influence of fluctuations in objective performance on task choice (resp. task ratings), entering objective performance as blockwise regressors. Regressors were z-scored to ensure comparability of regression coefficients. Participants were treated as a fixed effect in the regressions (due to few blocks per pairing per participant). In separate regression models we introduced individual self-esteem (Rosenberg score) as an additional regressor to examine if it could explain additional variance in task choice or task ratings.

Finally, to visualize the effects of learning duration, task choice frequencies were averaged across participants for each of the six possible pairings and the five possible learning durations (Fig. 4). To investigate whether learning duration had a significant influence on task choice, separate logistic regressions were performed on each of the six task pairings. Each model was specified as Task Choice $\sim \beta_{0}+\beta_{1} \times$ Learning Duration and participant was treated as a fixed effect (due to only one repetition of each learning block duration per participant). We additionally introduced individual selfesteem (Rosenberg score) as a predictor to examine if it influences task choices over and above learning duration, with a model specification similar as above: Task Choice $\sim \beta_{0}+\beta_{1} \times$ Learning Duration $+\beta_{2} \times$ Self-esteem score. Similarly, we examined whether learning duration influenced task ratings with similar models as for task choices, but with linear regression models instead of logistic regressions, because the dependent variable was continuous rather than dichotomous. Our dependent variable was the difference in task ratings between the two tasks of a block. 


\section{Acknowledgements}

MR is the beneficiary of a postdoctoral fellowship from the AXA Research Fund. MR's work is also supported by a department-wide grant from the Agence Nationale de la Recherche (ANR-17-EURE0017, EUR FrontCog). This work has received support under the program «Investissements d'Avenir» launched by the French Government and implemented by ANR (ANR-10-IDEX-0001-02 PSL).

G-J.W. was funded by the European Union's Horizon 2020 research and innovation program under the Marie Skłodowska-Curie grant agreement (No 707404) and the Sara van Dam z.l. Foundation, Royal Netherlands Academy of Arts \& Sciences.

SMF is supported by a Sir Henry Dale Fellowship jointly funded by the Wellcome Trust and Royal Society $(206648 / \mathrm{Z} / 17 / \mathrm{Z})$. The Wellcome Centre for Human Neuroimaging is supported by core funding from the Wellcome Trust (203147/Z/16/Z).

\section{Competing interests}

None of the authors declare any conflict of interest.

\section{Author contributions}

Conceptualization: M.R., G.J.W., S.M.F.; Data Collection: G.J.W., Methodology: M.R., G.J.W., S.M.F.; Formal Analysis: M.R.; Investigation: M.R. and G.J.W.; Writing - Original Draft: M.R.; Writing - Review \& Editing: G.J.W., S.M.F. and R.J.D.; Funding Acquisition: S.M.F. and R.J.D. 


\section{References}

1 Orth U, Robins RW, Roberts BW. Low self-esteem prospectively predicts depression in adolescence and young adulthood. Journal of Personality and Social Psychology 2008; 95: 695708.

2 Sowislo JF, Orth U. Does low self-esteem predict depression and anxiety? A meta-analysis of longitudinal studies. Psychological Bulletin 2013; 139: 213.

3 Bandura A. Self-efficacy: toward a unifying theory of behavioral change. Psychological Review 1977; 84: 191.

4 Di Paula A, Campbell JD. Self-esteem and persistence in the face of failure. Journal of Personality and Social Psychology 2002; 83: 711.

5 Seow TX, Rouault M, Gillan CM, Fleming SM. How local and global metacognition shape mental health. Biological Psychiatry 2021.

6 Will G-J, Rutledge RB, Moutoussis M, Dolan RJ. Neural and computational processes underlying dynamic changes in self-esteem. eLife 2017; 6: e28098.

7 Will G-J, Moutoussis M, Womack PM, Bullmore ET, Goodyer IM, Fonagy P et al. Neurocomputational mechanisms underpinning aberrant social learning in young adults with low self-esteem. Translational Psychiatry 2020; 10: 1-14.

8 Rouault M, Dayan P, Fleming SM. Forming global estimates of self-performance from local confidence. Nature Communications 2019; 10: 1141.

9 Rouault M, Fleming SM. Formation of global self-beliefs in the human brain. Proceedings of the National Academy of Sciences 2020; 117: 27268-27276.

10 Stephan KE, Manjaly ZM, Mathys CD, Weber LA, Paliwal S, Gard T et al. Allostatic SelfEfficacy: A Metacognitive Theory of Dyshomeostasis-Induced Fatigue and Depression. Front Hum Neurosci 2016; 10: 550.

11 Vinckier F, Rigoux L, Oudiette D, Pessiglione M. Neuro-computational account of how mood fluctuations arise and affect decision making. Nature Communications 2018; : 1-12.

12 Rouault M, McWilliams A, Allen MG, Fleming SM. Human Metacognition Across Domains: Insights from Individual Differences and Neuroimaging. Personality Neuroscience 2018; 1: e1713.

13 Baumeister RF, Campbell JD, Krueger JI, Vohs KD. Does high self-esteem cause better performance, interpersonal success, happiness, or healthier lifestyles? Psychological science in the public interest 2003; 4: 1-44.

14 Hu X, Zheng J, Su N, Fan T, Yang C, Yin Y et al. A Bayesian inference model for metamemory. Psychological Review 2021.

15 Desender K, Sasanguie D. Math anxiety relates positively to metacognitive insight into mathematical decision making. Psychological Research 2021; : 1-13.

16 Huguet P, Régner I. Counter-stereotypic beliefs in math do not protect school girls from stereotype threat. Journal of Experimental Social Psychology 2009; 45: 1024-1027. 
17 Gajdos T, Régner I, Huguet P, Hainguerlot M, Vergnaud J-C, Sackur J et al. Does social context impact metacognition? Evidence from stereotype threat in a visual search task. PLoS ONE 2019; 14: e0215050.

18 Moses-Payne ME, Rollwage M, Fleming SM, Roiser JP. Postdecision Evidence Integration and Depressive Symptoms. Frontiers in psychiatry 2019; 10: 639.

19 Brown JD. High self-esteem buffers negative feedback: Once more with feeling. Cognition and Emotion 2010; 24: 1389-1404.

20 David AS, Bedford N, Wiffen B, Gilleen J. Failures of metacognition and lack of insight in neuropsychiatric disorders. Philosophical Transactions of the Royal Society B: Biological Sciences 2012; 367: 1379-1390.

21 Hoven M, Lebreton M, Engelmann JB, Denys D, Luigjes J, van Holst RJ. Abnormalities of confidence in psychiatry: an overview and future perspectives. Translational Psychiatry 2019; 9: $1-18$.

22 Rouault M, Seow T, Gillan CM, Fleming SM. Psychiatric Symptom Dimensions Are Associated With Dissociable Shifts in Metacognition but Not Task Performance. Biological Psychiatry 2018.

23 Koban L, Schneider R, Ashar YK, Andrews-Hanna JR, Landy L, Moscovitch DA et al. Social anxiety is characterized by biased learning about performance and the self. Emotion 2017; 17: 1144 .

24 Aarts K, Pourtois G. Anxiety disrupts the evaluative component of performance monitoring: An ERP study. Neuropsychologia 2012; 50: 1286-1296.

25 Lee AL, de Gardelle V, Mamassian P. Global visual confidence. Psychonomic Bulletin \& Review 2021;: $1-10$.

26 Sherman M, Seth A. Effects of expected task difficulty on metacognitive confidence and multitasking. 2021.

27 Weil LG, Fleming SM, Dumontheil I, Kilford EJ, Weil RS, Rees G et al. The development of metacognitive ability in adolescence. Consciousness and Cognition 2013; 22: 264-271.

28 Seow TXF, Rouault M, Gillan CM, Fleming SM. How local and global metacognition shape mental health. Biological Psychiatry 2021.

29 Kiebel SJ, Daunizeau J, Friston KJ. A hierarchy of time-scales and the brain. PLoS Comput Biol 2008; 4: e1000209.

30 Rosenberg M. Rosenberg self-esteem scale (RSE). Acceptance and commitment therapy Measures package 1965; 61: 18.

31 Kiddle B, Inkster B, Prabhu G, Moutoussis M, Whitaker KJ, Bullmore ET et al. Cohort profile: The NSPN 2400 cohort: A developmental sample supporting the Wellcome Trust NeuroScience in Psychiatry Network. International journal of epidemiology 2018; 47: 18-19g. 


\section{Supplementary Material}

\section{Supplementary Figures}
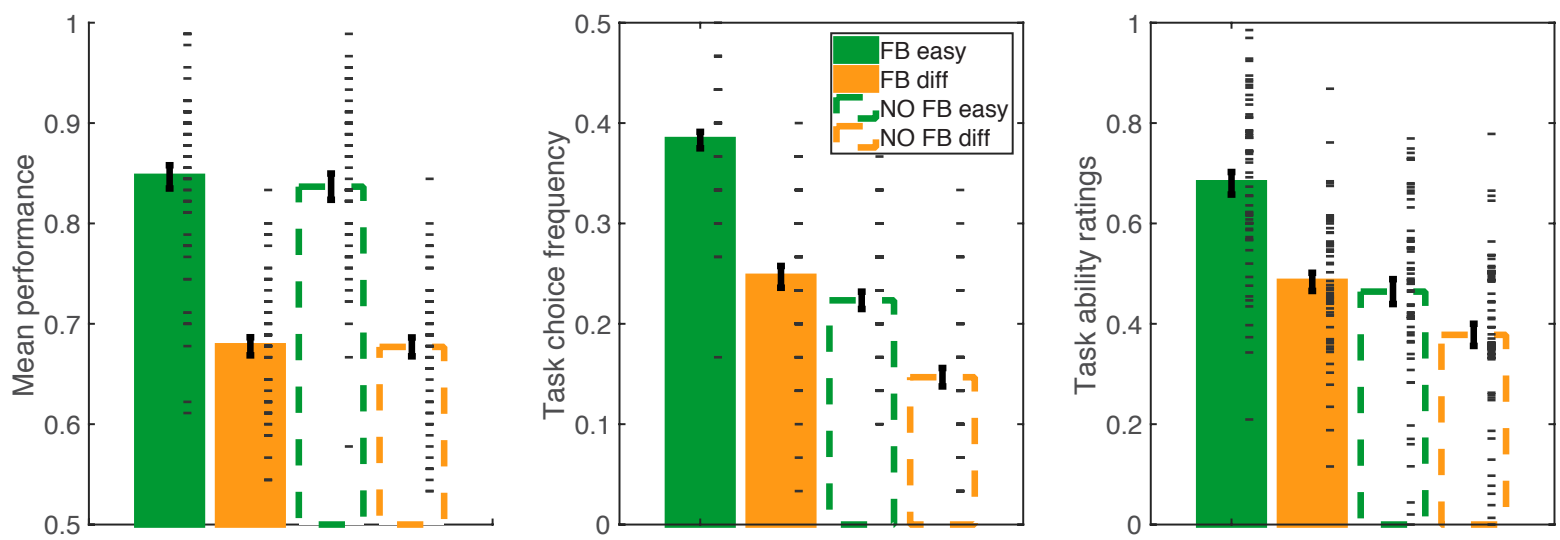

Figure S1. Behavioral dissociation between objective performance (left panel) and self-performance estimates, measured as end-of-block task choices (middle panel) and task ratings (right panel). ). Green (resp. orange) indicates easy (resp. difficult) tasks. Dotted lines (resp. full bars) indicate tasks with no feedback (resp. with feedback). Error bars indicate S.E.M. across participants (N=57) with black ticks indicating individual data points. See also Results. Note that task ratings are between 0 and 1 due to non-parametric z-score within participant. 


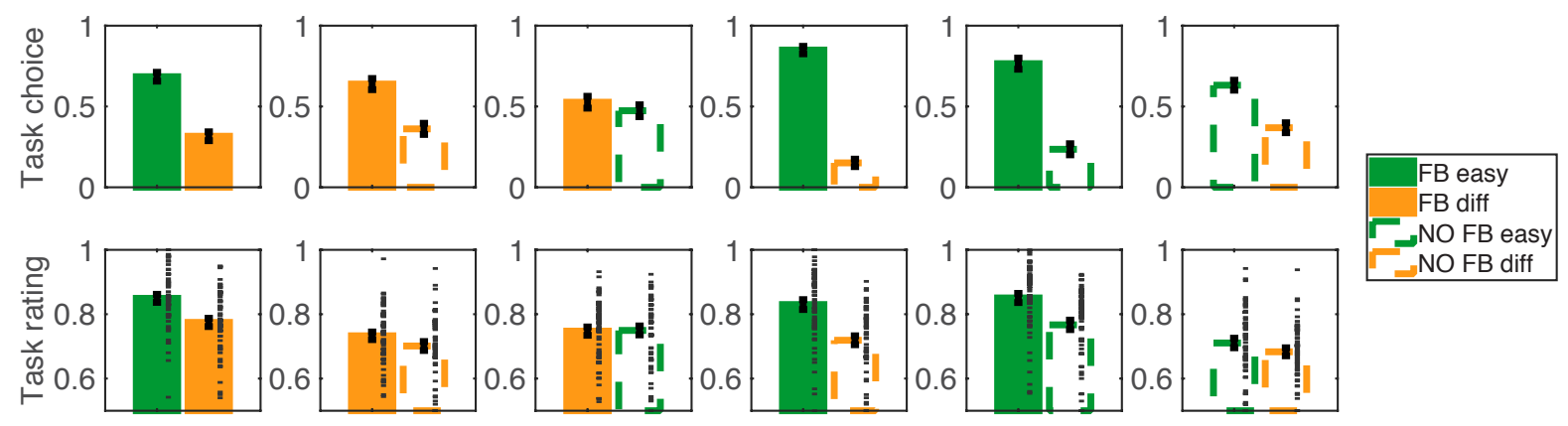

Figure S2. Self-performance estimates assessed at the end of blocks via task choices (upper panels) and task ratings (lower panels). Green (resp. orange) indicates easy (resp. difficult) tasks. Dotted lines (resp. full bars) indicate tasks with no feedback (resp. with feedback). Error bars indicate S.E.M. across participants $(N=57)$ with black ticks indicating individual data points. 


\section{Supplementary Tables}

Within Subjects Effects

\begin{tabular}{lcrrrr}
\hline & Sum of Squares & df & \multicolumn{1}{c}{ Mean } & \multicolumn{1}{c}{ Square } & \multicolumn{1}{c}{$\mathrm{p}$} \\
\hline Difficulty & 1.543 & 1 & 1.543 & $472.719<.001$ \\
Difficulty $\times$ Self-Esteem & 0.017 & 1 & 0.017 & 5.174 & 0.027 \\
Residual & 0.179 & 55 & 0.003 & & \\
Feedback & 0.002 & 1 & 0.002 & 0.622 & 0.434 \\
Feedback $\times$ Self-Esteem & $8.636 \mathrm{e}-4$ & 1 & $8.636 \mathrm{e}-4$ & 0.344 & 0.560 \\
Residual & 0.138 & 55 & 0.003 & & \\
Difficulty $\times$ Feedback & 0.001 & 1 & 0.001 & 0.630 & 0.431 \\
Difficulty $\times$ Feedback $\times$ Self-Esteem & $6.326 \mathrm{e}-4$ & 1 & $6.326 \mathrm{e}-4$ & 0.342 & 0.561 \\
Residual & 0.102 & 55 & 0.002 & & \\
\hline
\end{tabular}

Note. Type III Sum of Squares

Between Subjects Effects

\begin{tabular}{lcccccc}
\hline & $\begin{array}{c}\text { Sum of } \\
\text { Squares }\end{array}$ & df & Mean Square & F & $p$ \\
\hline Self-Esteem & 0.032 & 1 & 0.032 & 1.675 & 0.201 & \\
Residual & 1.045 & 55 & 0.019 & & & \\
\hline & Note. & & & & &
\end{tabular}

Note. Type III Sum of Squares

Table S1: A $2 \times 2 \times 2$ repeated measures ANOVA on performance with Difficulty (Easy, Difficult) and Feedback (Feedback, No Feedback) as within-subject factors and Self-Esteem (high, low) as a between-subject factor revealed a main effect of task difficulty on performance, as expected. No other main effects or interactions were significant (see Results). 


\begin{tabular}{|c|c|c|c|c|c|}
\hline & Sum of Squares & df & $\begin{array}{l}\text { Mean } \\
\text { Square }\end{array}$ & $F$ & $p$ \\
\hline Difficulty & 3.819 & 1 & 3.819 & 108.779 & $<.001$ \\
\hline Difficulty $\times$ Self-Esteem & 0.035 & 1 & 0.035 & 0.987 & 0.325 \\
\hline Residual & 1.931 & 55 & 0.035 & & \\
\hline Feedback & 5.431 & 1 & 5.431 & 93.775 & $<.001$ \\
\hline Feedback $\times$ Self-Esteem & 0.004 & 1 & 0.004 & 0.073 & 0.788 \\
\hline Residual & 3.185 & 55 & 0.058 & & \\
\hline Difficulty $\times$ Feedback & 0.128 & 1 & 0.128 & 3.808 & 0.056 \\
\hline Difficulty $\times$ Feedback $\times$ Self-Esteem & 0.004 & 1 & 0.004 & 0.130 & 0.720 \\
\hline Residual & 1.846 & 55 & 0.034 & & \\
\hline
\end{tabular}

Note. Type III Sum of Squares

Between Subjects Effects

\begin{tabular}{lrrrrrl}
\hline & $\begin{array}{c}\text { Sum of } \\
\text { Squares }\end{array}$ & df & Mean Square & F & $p$ \\
\hline Self-Esteem & $2.968 \mathrm{e}-4$ & 1 & $2.968 \mathrm{e}-$ & 0.295 & 0.589 & \\
Residual & 0.055 & 55 & 0.001 & & & \\
\hline
\end{tabular}

Note. Type III Sum of Squares

Table S2: A $2 \times 2 \times 2$ repeated measures ANOVA on end-of-block task choices with Difficulty (Easy, Difficult) and Feedback (Feedback, No Feedback) as within-subject factors and Self-Esteem (high, low) as a between-subject factor revealed a main effect of Difficulty and Feedback factors on task choice. No other main effects or interactions were significant (see Results). 


\begin{tabular}{lrrrrr}
\hline & Sum of Squares & df Mean Square & \multicolumn{1}{c}{$\mathrm{F}$} & $\mathrm{p}$ \\
\hline Difficulty & 2521.945 & 1 & 2521.945 & $211.732<.001$ \\
Difficulty $\times$ Self-Esteem & 16.407 & 1 & 16.407 & 1.377 & 0.246 \\
Residual & 655.106 & 55 & 11.911 & & \\
Feedback & 3259.154 & 1 & 3259.154 & $139.926<.001$ \\
Feedback $\times$ Self-Esteem & 11.768 & 1 & 11.768 & 0.505 & 0.480 \\
Residual & 1281.056 & 55 & 23.292 & & \\
Difficulty $\times$ Feedback & 361.370 & 1 & 361.370 & $35.629<.001$ \\
Difficulty $\times$ Feedback $\times$ Self-Esteem & 0.880 & 1 & 0.880 & 0.087 & 0.769 \\
Residual & 557.838 & 55 & 10.143 & & \\
\hline
\end{tabular}

Note. Type III Sum of Squares

Between Subjects Effects

\begin{tabular}{|c|c|c|c|c|}
\hline & Sum of Squares df & an Square & $F$ & $p$ \\
\hline$\overline{\text { elf- }}$ & 1581.9841 & 1581.984 & 5.9150 & .018 \\
\hline esic & 14709.83755 & 267.452 & & \\
\hline
\end{tabular}

Note. Type III Sum of Squares

Table S3: A $2 \times 2 \times 2$ repeated measures ANOVA on end-of-block task ratings with Difficulty (Easy, Difficult) and Feedback (Feedback, No Feedback) as within-subject factors and Self-Esteem (high, low) as a between-subject factor revealed a main effect of Difficulty and Feedback factors on task choice, with a significant interaction between these factors, together with a main effect of Self-Esteem. No other main effects or interactions were significant (see Results). 